\title{
A NEW PROOF OF E. CARTAN'S THEOREM ON THE TOPOLOGY OF SEMI-SIMPLE GROUPS
}

\author{
GEORGE DANIEL MOSTOW ${ }^{1}$
}

1. Introduction. E. Cartan has proved ${ }^{2}$ that a connected semisimple Lie group is topologically the direct product of a compact subgroup and a Euclidean space. Cartan first proved this theorem in 1927 by a reduction to special cases, and not until 1929 did he free his proof from the consideration of special cases. As a result Cartan's proof is diffused among several journals. Moreover, Cartan employs in an essential way the theory of symmetric Riemannian spaces and makes use of a result whose proof seems to be lacking (Ann. École Norm. loc. cit. p. $367, \S 20$ ).

In this paper there will be given a more direct proof which eliminates the use of symmetric Riemannian spaces. The author wishes to acknowledge his debt to Professor C. Chevalley who suggested in an oral communication Lemma 1.4 below and to whom a proof of Theorem 1, essentially the same as the one given here, was known.

2. Definitions and preliminaries. Let $G$ be a semi-simple Lie algebra over a field $K$ of characteristic zero. Let ad $g$ denote the linear transformation $x \rightarrow[g, x]$, where $x, g \in G$. By a Cartan subalgebra is meant a subalgebra $\mathfrak{F}$ of $G$ maximal with respect to properties (1) $\mathfrak{H}$ is abelian, that is, $[\mathfrak{H C}, \mathfrak{H C}]=0$; and (2) $H \in \mathcal{H C}$ implies ad $H$ is semisimple, that is, its minimal equation has no repeated factor. It is a theorem that a Cartan subalgebra is a maximal abelian subalgebra (but not conversely).

Let $G$ be a semi-simple Lie algebra, that is, $G$ contains no abelian ideal, and let $\mathfrak{H C}$ be any Cartan subalgebra. Assume that the base field $K$ is algebraically closed. A nonzero linear function $\alpha$ defined on $\mathcal{H}$ is called a "root" if and only if there exists an $X$ in $G$ such that $[H, X]$ $=\alpha(H), X$ for all $H \in \mathfrak{F}$. Any nonzero $Y$ in $G$ with this property is said to "belong to $\alpha . "$

The following is known: ${ }^{3}$

R1. If $X_{\alpha}, X_{\beta}$ belong to $\alpha, \beta$ respectively, then $\left[X_{\alpha}, X_{\beta}\right]$ belongs

Presented to the Society, September 10, 1948; received by the editors September 2, 1948.

${ }^{1}$ O.N.R. Fellowship holder, 1948-1949.

2 Ann. Ecole Norm. vol. 44 (1927) p. 367, cf. Bull. Soc. Math. France vol. 55 (1927) pp. 122-125, J. Math. Pures Appl. (9) vol. 8 (1929) pp. 24-27.

${ }^{3}$ Cf. H. Weyl, Continuous groups, vol. 2, Institute for Advanced Study Notes, 1935, pp. 69-87. 
to $\alpha+\beta$ or is zero according as $\alpha+\beta$ is a root or not.

$\mathrm{R} 2$. The linear subspace of elements of $G$ belonging to a root is one-dimensional.

Notation. " $X_{\alpha}$ " denotes an element belonging to the root $\alpha$.

Clearly any collection $\left\{X_{\alpha}\right\}$ with distinct subscripts are linearly independent over $K \bmod \mathfrak{H C}$ and $\left\{\mathfrak{F}, X_{\alpha} \mid\right.$ all roots $\left.\alpha\right\}$ spans $G$.

Suppose $\alpha, \beta$ are roots. Define

$$
\begin{aligned}
k(\alpha, \beta) & =\max 1(\beta-\nu \alpha \text { is a root, } 0 \leqq \nu \leqq 1 ; \nu, 1 \text { integers }), \\
k^{\prime}(\alpha, \beta) & =\max \mathrm{l}(\beta+\nu \alpha \text { is a root, } 0 \leqq \nu \leqq 1 ; \nu, 1 \text { integers }) .
\end{aligned}
$$

Define $B(X, Y)=$ Trace $(\operatorname{ad} X$ ad $Y) . B$ is called the "fundamental bilinear form."

R3. $B(X, Y)$ is nondegenerate on $G$ and on $\mathcal{H}$. If $\alpha+\beta \neq 0$, $B\left(X_{\alpha}, X_{\beta}\right)=0 . B\left(H, X_{\alpha}\right)=0$, if $H \in \mathcal{H C}$, for all roots $\alpha$.

Define $H_{\alpha}$ as the element of $\mathcal{H}$ such that $B\left(H, H_{\alpha}\right)=\alpha(H)$ for all $H \in H$.

R4.

$$
\begin{aligned}
\beta\left(H_{\alpha}\right) & =\frac{k(\alpha, \beta)-k^{\prime}(\alpha, \beta)}{2} \alpha\left(H_{\alpha}\right), \\
\frac{4}{\alpha\left(H_{\alpha}\right)} & =\sum_{\beta}\left[k(\alpha, \beta)-k^{\prime}(\alpha, \beta)\right]^{2}, \\
{\left[X_{\alpha}, X_{-\alpha}\right] } & =B\left(X_{\alpha}, X_{-\alpha}\right) H_{\alpha} .
\end{aligned}
$$

For each root $\alpha$ select an $X_{\alpha}$. Define $C_{\alpha \beta}$ by the relations

$$
\begin{aligned}
{\left[X_{\alpha}, X_{\beta}\right] } & =C_{\alpha, \beta} X_{\alpha+\beta} \text { if } \alpha+\beta \text { is a root, } \\
C_{\alpha, \beta} & =0 \text { if } \alpha+\beta \text { is not a root. }
\end{aligned}
$$

R5. $C_{\alpha, \beta} C_{-\alpha,-\beta}=-2^{-1} k^{\prime}(\alpha, \beta)[k(\alpha, \beta)+1] \alpha\left(H_{\alpha}\right)$.

R6. $\left\{X_{\alpha} \mid\right.$ all roots $\left.\alpha\right\}$ may be so chosen that $\left[X_{\alpha}, X_{-\alpha}\right]=H_{\alpha}$ and $C_{\alpha, \beta}=-C_{-\alpha,-\beta}$. Here $B\left(X_{\alpha}, X_{-\alpha}\right)=1$ and $C_{\alpha, \beta}$ is real if $K$ is the complex numbers.

3. An algebraic theorem. We shall now deal exclusively with Lie algebras over the real and complex number fields $R$ and $C$ respectively.

Let $G$ be a Lie algebra over $C$. By a real form of $G$ is meant a real linear subspace which (1) is closed under the formation of brackets; (2) spans $G$; and (3) has a base which is linearly independent over $C$. If $G$ is a Lie algebra over $R$, by the complexification of $G$ is meant the augmented linear space over $C$ obtained by augmenting $R$ to $C$.

Let $G$ be a Lie algebra over $C$ and let $G_{R}$ be a real form. Consider the real-linear transformation $\theta$ of $G$ (considered as a linear space 
over $R)$ :

$$
\theta: \quad X+(-1)^{1 / 2} Y \rightarrow X-(-1)^{1 / 2} Y, \text { where } X, Y \in G_{R} .
$$

Definition. $\theta$ is called the "conjugation of $G$ with respect to $G_{R}$." Note that $G_{R}$ is the set of elements which are invariant under $\theta$.

Note too that $\theta\left(\left[X+(-1)^{1 / 2} Y, \quad U+(-1)^{1 / 2} V\right]\right)=\theta\left(\left(\left[\begin{array}{ll}X, & U\end{array}\right]\right.\right.$ $\left.-[Y, V])+(-1)^{1 / 2}([X, V]+[Y, U])\right)=[X, U]-[Y, V]-(-1)^{1 / 2}$ $\cdot([X, V)+[Y, V])=\left[X-(-1)^{1 / 2} Y, U-(-1)^{1 / 2} V\right]$ if $X, Y, U$, $V \in G_{R}$. Thus $\theta([X, Y)=[\theta(X), \theta(Y)]$ if $X, Y \in G$.

Definition. A compact form of a complex Lie algebra is a real form on which the fundamental bilinear form is negative definite.

This section is devoted to the proof of the following theorem.

TheOREM 1. Let $G$ be a semi-simple Lie algebra over $R$, let $G_{C}$ denote its complexification, and let $\theta$ denote the conjugation of $G_{c}$ with respect to $G$. Then $\theta$ leaves invariant a compact real form $G_{K}$.

Notation. " $X$ " denotes $\theta(X)$, where $X \in G c$.

Clearly $G_{C}$ is a semi-simple Lie algebra. Assume that for each root $\alpha$ an $X_{\alpha}$ is so chosen as to make $\left\{X_{\alpha} \mid\right.$ all roots $\left.\alpha\right\}$ satisfy the condition in R6 above.

Lemma 1.1. Let $\mathfrak{x}$ be a Cartan subalgebra of $G$ and let $\mathcal{H}_{C}$ denote the complexification of $\mathfrak{H C}^{\mathrm{C}} \mathfrak{H}_{C}$ is a Cartan subalgebra of $G c$. Moreover to each root $\alpha$ there corresponds a root $\alpha^{\prime}$ such that

(1) $\alpha^{\prime}(H)=\bar{\alpha}(H)$ for $H \in \mathcal{H}$,

(2) $\left[H, X_{\alpha}^{\prime}\right]=\alpha^{\prime}(H) X_{\alpha}^{\prime}$ for $H \in \mathfrak{H C}_{C}$,

(3) if $\alpha, \beta, \alpha+\beta$ are roots, then $\alpha^{\prime}+\beta^{\prime}$ is a root and $(\alpha+\beta)^{\prime}=\alpha^{\prime}+\beta^{\prime}$.

Proof. Inasmuch as $\left\{\mathrm{ad} H \mid H \in \mathcal{H}_{C}\right\}$ is a commutative set of linear transformations and is spanned by semi-simple transformations, and inasmuch as $C$ is algebraically closed, all the elements of $\left\{\right.$ ad $\left.H \mid H \in \mathfrak{H}_{C}\right\}$ are semi-simple.

Suppose $X+(-1)^{1 / 2} Y$, where $X, Y \in G$, is an element of a Cartan subalgebra of $G_{C}$ which includes $\mathfrak{H}_{C}$. Then $[H, X]=[H, Y]=0$ for $H \in \mathcal{H}$. Fe being a maximal abelian subalgebra, $X$ and $Y$ must be in $\mathfrak{H C}$ so that $X+(-1)^{1 / 2} Y \in \mathcal{H C}_{C}$. It follows that $\mathfrak{H}_{C}$ is a Cartan subalgebra of $G c$.

From

$$
\theta([X, Y])=\left[X^{\prime}, Y^{\prime}\right]
$$

and

$$
\theta(\alpha X)=\bar{\alpha} X^{\prime} \quad \text { if } X, Y \in G c
$$


we infer that

$$
\left[H, X_{\alpha}^{\prime}\right]=\bar{\alpha}(H) X_{\alpha}^{\prime}, \quad \text { if } H \in \mathcal{H} .
$$

Lemma 1.1 follows from this.

LEMMA 1.2. $C_{\alpha, \beta}= \pm C_{\alpha^{\prime}, \beta^{\prime}}$.

Proof. $-C_{\alpha, \beta}^{2}=-2^{-1} k^{\prime}(\alpha, \beta)[k(\alpha, \beta)+1] \alpha\left(H_{\alpha}\right)$. From Lemma 1.1 it follows directly that

$$
k(\alpha, \beta)=k\left(\alpha^{\prime}, \beta^{\prime}\right), k^{\prime}(\alpha, \beta)=k^{\prime}\left(\alpha^{\prime}, \beta^{\prime}\right) .
$$

Since

$$
\frac{4}{\alpha\left(H_{\alpha}\right)}=\sum_{\beta}\left[k(\alpha, \beta)-k^{\prime}(\alpha, \beta)\right]^{2}
$$

it follows that $C_{\alpha, \beta}= \pm C_{\alpha^{\prime}, \beta^{\prime}}$.

LEMMA 1.3. $H_{\alpha^{\prime}}=H_{\alpha}^{\prime}$.

Proof. Since the fundamental bilinear form $B$ is real on $G$, $B\left(X+(-1)^{1 / 2} Y, \quad U+(-1)^{1 / 2} V\right)$ is the complex conjugate of $B\left(X-(-1)^{1 / 2} Y, U-(-1)^{1 / 2} V\right)$ if $X Y \in G$, that is, $B\left(Z^{\prime}, W^{\prime}\right)$ is the complex conjugate of $B(Z, W)$ if $Z, M \in G_{C}$. Hence $B\left(H, H_{\alpha}^{\prime}\right)$ is the complex conjugate of $B\left(H, H_{\boldsymbol{\alpha}}\right)$ is the complex conjugate of $\alpha(H)=\alpha^{\prime}(H)$ if $H \in \mathcal{H C}$. Consequently $B\left(H, H_{\alpha}^{\prime}\right)=\alpha^{\prime}(H)$ for all $H \in \mathcal{H}_{C}$ and thus by definition $H_{\alpha^{\prime}}=H_{\alpha}^{\prime}$.

Let $u_{\alpha}$ be defined by the relation

$$
X_{\alpha}^{\prime}=u_{\alpha} X_{\alpha^{\prime}} \quad(\text { all roots } \alpha) .
$$

Since $C_{\alpha, \beta}$ are real, $u_{\alpha} u_{\beta}= \pm u_{\alpha+\beta}$ if $\alpha, \beta, \alpha+\beta$ are roots. Also $\bar{u}_{\alpha} u_{\alpha^{\prime}}=1$, $u_{\alpha} u_{-\alpha}=1$.

LEMmA 1.4. The choice of $\left\{X_{\alpha} \mid\right.$ all roots $\left.\alpha\right\}$ can be modified without changing the constants of structure $C_{\alpha, \beta}$ so as to make $\left|u_{\alpha}\right|=1$ for all roots $\alpha$.

Proof. ${ }^{4}$ Let $r_{\alpha}=\left|u_{\alpha}\right|^{-1 / 2}$ and let $Y_{\alpha}=r_{\alpha} X_{\alpha}$ for all roots $\alpha$. Then $r_{\alpha} r_{\beta}=r_{\alpha+\beta}$ and $r_{\alpha} r_{-\alpha}=1$ if $\alpha, \beta, \alpha+\beta$ are roots. It follows immediately that if we replace the $\left\{X_{\alpha}\right\}$ by $\left\{Y_{\alpha}\right\}$, then the constants of structure are unchanged. Moreover

$$
\theta\left(Y_{\alpha}\right)=v_{\alpha} Y_{\alpha^{\prime}}
$$

where $\left|v_{\alpha}\right|=\left|r_{\alpha} u_{\alpha^{\prime}} r_{\alpha^{\prime}}^{-1}\right|=\left|u_{\alpha}\right|^{-1 / 2} \cdot\left|u_{\alpha}\right| \cdot\left|u_{\alpha^{\prime}}\right|^{1 / 2}=1$. The lemma is

${ }^{4}$ The proof given here is a simplification, pointed out by Dr. Harish-Chandra, of the author's proof. 
thus proved.

We assume now that $G_{c}$ has a base $\left\{H_{\alpha_{i}}, X_{\alpha} \mid\right.$ all roots $\left.\alpha\right\}$ such that $\left|u_{\alpha}\right|=1$. Since $u_{\alpha} u_{-\alpha}=1$, it follows that $u_{-\alpha}=\bar{u}_{\alpha}$.

Proof of TheOREM 1. Let $\mathcal{F}_{0}$ denote the real linear space spanned by $\left\{H_{\alpha} \mid\right.$ all roots $\left.\alpha\right\}$ and let $G_{K}$ denote the real linear spaced spanned by $\left\{(-1)^{1 / 2} \mathcal{F}_{0}, z_{\alpha} X_{\alpha}-\bar{z}_{\alpha} X_{-\alpha}\right\}$ all roots $\alpha, z_{\alpha}$ assuming all complex values $\}$. Since $C_{\alpha, \beta}$ is real and $C_{-\alpha,-\beta}=-C_{\alpha, \beta}$, it is readily seen that $G_{K}$ is closed under formation of brackets. Moreover $\left\{H_{1}, \cdots, H_{l}\right.$, $X_{\alpha}-X_{-\alpha},(-1)^{1 / 2}\left(X_{\alpha}+X_{-\alpha}\right) \mid$ all pairs of roots $\alpha$ and $-\alpha ; H_{1}, \cdots, H_{l}$ base for $\left.(-1)^{1 / 2} \mathcal{H}_{0}\right\}$ is a base for $G_{c}$ with respect to complex numbers. It follows that $G_{K}$ is a real form of $G_{c}$. If $Z \in G_{K}$, then $Z=(-1)^{1 / 2} H$ $+\sum_{\alpha}\left(z_{\alpha} X_{\alpha}-\bar{z}_{\alpha} X_{-\alpha}\right)$, where $H \in \mathcal{H}_{0}$. Thus

$$
\begin{aligned}
\theta(Z) & =-(-1)^{1 / 2} H^{\prime}+\sum_{\alpha}\left(\bar{z}_{\alpha} u_{\alpha} X_{\alpha^{\prime}}-z_{\alpha} u_{-\alpha} X_{-\alpha^{\prime}}\right) \\
& =-(-1)^{1 / 2} H^{\prime}+\sum_{\alpha}\left(\bar{z}_{\alpha} u_{\alpha} X_{\alpha^{\prime}}-z_{\alpha} \bar{u}_{\alpha} X_{-\alpha^{\prime}}\right)
\end{aligned}
$$

where $H^{\prime} \in \mathcal{H}_{0}$. Hence $G_{k}$ is invariant under the conjugation $\theta$. Finally, $G_{K}$ is a compact real form. For if $Z \in G_{K}$, then

$$
Z=(-1)^{1 / 2} H+\sum_{\alpha}\left(z_{\alpha} X_{\alpha}-\bar{z}_{\alpha} X_{-\alpha}\right), \quad H \in \mathfrak{H}_{0} .
$$

By R3, $B(Z, Z)=-B(H, H)-\sum_{\alpha} z_{\alpha} \bar{z}_{\alpha}$. But

$$
B(H, H)=\operatorname{Tr}(\operatorname{ad} H)(\operatorname{ad} H)=\sum_{\alpha} \alpha^{2}(H) \geqq 0
$$

with inequality holding only if $H=0$, since all roots assume real values on $\mathfrak{H}_{0}$ (cf. R4). Hence $B(Z, Z)<0$ if $Z \neq 0$. Thus $G_{K}$ is a compact real form invariant under the conjugation $\theta$.

4. The theorem. Let $G$ be a connected real semi-simple Lie group, $G$ its Lie algebra, and let ad $G$ denote its adjoint group, that is, the Lie group of autmorphisms of $G$ induced by the inner automorphisms of $G$. Let $G_{C}$ denote the complexification of $G, \theta$ the conjugation of $G_{c}$ with respect to $G, G_{K}$ a real compact form of $G_{C}$ which is invariant under $\theta$. ad $G_{C}$, being a subalgebra of the Lie algebra of all linear transformations of $G_{C}$, determines a Lie subgroup $G_{C}^{*}$ of the Lie group of all nonsingular linear transformations on $G_{c}$. To $G$ there corresponds a real linear subspace $G^{*} C$ ad $G_{C}$ under the correspondence:

$$
X \in G \leftrightarrow \operatorname{ad} X \in \operatorname{ad} G c .
$$

To $G_{K}$ there corresponds, under the correspondence

$$
X \in G_{K} \leftrightarrow \operatorname{ad} X \in \operatorname{ad} G_{c},
$$


a real linear subspace $G_{K}^{*} \subset$ ad $G_{c}$. Regarding ad $G_{c}$ as a Lie algebra over the reals (that is, regard ad $G_{c}$ as a linear space over the reals; since the bracket operation satisfies the identities of a Lie algebra, ad $G_{C}$ is a real Lie algebra) $G^{*}$ and $G_{K}^{*}$ are then subalgebras and to them correspond subgroups $G^{*}$ and $G_{K}^{*}$ of $G_{C}^{*}$. Clearly $G^{*} \cong$ ad $G$ topologically as well as algebraically.

Lemma 2.1. $G=G \cap G_{K}+G \cap(-1)^{1 / 2} G_{K}$ (directly), that is, $G$ has a base $\left\{X_{1}, \cdots, X_{P},(-1)^{1 / 2} X_{P+1}, \cdots,(-1)^{1 / 2} X_{r}\right\}$ with $X_{i} \in G_{K}$ $(i=1,2, \cdots, r)$. Moreover, there is base $\mathcal{C}$ for $G_{c}$ relative to which the matrix of ad $X$ is real skew-symmetric for all $X \in G_{K}$.

Proof. Since the real form $G_{K}$ is invariant under $\theta$ and since $\theta^{2}$ is the identity, there is a base $\left\{X_{1}, \cdots, X_{p}, X_{p+1}, \cdots, X_{r}\right)$ of $G_{K}$ such that $\theta\left(X_{i}\right)=X_{i}(i=1, \cdots, p)$ and $\theta\left(X_{j}\right)=-X_{j}(j=p+1, \cdots, r)$. Inasmuch as $\theta\left((-1)^{1 / 2} X_{j}\right)=(-1)^{1 / 2} X_{j}(j=p+1, \cdots, r)$, one infers that $(-1)^{1 / 2} X_{j} \in G \quad(j=p+1, \cdots, r)$. Clearly $\left\{X_{1}, \cdots, X_{P}\right.$; $\left.(-1)^{1 / 2} X_{P+1}, \cdots,(-1)^{1 / 2} X_{r}\right\}$ is a base for $G$, that is, $G=G \cap G_{K}$ $+G \cap(-1)^{1 / 2} G_{K}$ (directly).

The fundamental bilinear form being negative definite on the compact real form $G_{K}$, a base $\mathcal{C}=\left\{Z_{1}, \cdots, Z_{r}\right\}$ can be selected for $G_{C}$ such that $Z_{i} \in \mathcal{G}_{K}(i=1, \cdots, r)$ and such that $B(X, Y)=-\sum_{i=1}^{r} x_{i} y_{i}$ if $X=\sum_{i=1}^{r} x_{i} Z_{i}, Y=\sum_{i=1}^{r} y_{i} Z_{i}$. By the Jacobian identities, ad $[X, Y]=\operatorname{ad} Y$ ad $X-\operatorname{ad} X$ ad $Y$. Hence

$$
\begin{aligned}
B(\operatorname{ad} X(Y), Z) & +B(Y, \text { ad } X(Z)) \\
= & \text { Trace ad }[X, Y] \operatorname{ad} Z+\text { Trace ad } Y \text { ad }[X, Z] \\
= & \text { Trace }(\operatorname{ad} X)(\operatorname{ad} Y \text { ad } Z)-\text { Trace }(\operatorname{ad} Y \operatorname{ad} Z)(\operatorname{ad} X) \\
= & 0 .
\end{aligned}
$$

It follows that, relative to $\mathcal{C}$, the matrix of ad $X$ is real skew-symmetric if $X \in G_{K}$.

Definition. Let $v$ be a linear space over a field $K$ and let $\varepsilon$ denote the group of nonsingular linear transformations on $\mathcal{V}$. Let $\mathcal{C}$ denote a base for $\mathcal{V}$ and $T_{M}$ the matrix of $T \in \mathcal{E}$ relative to $\mathcal{C}$. If $H \subset \mathcal{E}$, $\left\{T_{M} \mid T \in H\right\}$ is denoted by $H_{M}$. A subgroup $G$ of $\mathcal{E}$ is called "algebraic relative to $\mathcal{C}$ " if $G_{M}$ is the intersection of an algebraic subvariety of the space of matrices of all linear transformations of $V$ with $\varepsilon_{M}$.

It is to be noted that if a group is algebraic relative to one base for $V$, then it is algebraic relative to any other base. Hence one can use simply the term "algebraic."

In our usage of algebraic groups, the field $K$ will always be the reals. 
Definition. Let $G$ be a Lie algebra over the complex numbers and $G_{0}$ a real form of $G$. The set of all automorphisms of $G$ is called "aut G." The subset of aut $G$ keeping $G_{0}$ invariant is called "aut $G_{0}$."

Let $T$ be a complex-linear transformation of $G_{c}$. If $G_{c}$ is regarded as a linear space over the reals, then the transformation $T$ is a fortiori real-linear.

Lemma 2.2. Let $G_{c}$ be regarded as a linear space over the reals. Then aut $G_{c}$, aut $G_{\text {, and }}$ aut $\mathcal{G}_{K}$ are algebraic groups.

Proof. Let $\mathcal{D}=\left\{X_{1}, \cdots, X_{r},(-1)^{1 / 2} X_{1}, \cdots,(-1)^{1 / 2} X_{r}\right\}$ be a base for $G_{C}$ considered as a linear space over the reals. Let $X_{r+k}$ denote $(-1)^{1 / 2} X_{k}(k=1, \cdots, r)$. Let $\iota$ denote the real-linear transformation determined by the mapping

$$
\begin{aligned}
X_{k} & \rightarrow(-1)^{1 / 2} X_{k}, \\
(-1)^{1 / 2} X_{k} & \rightarrow-X_{k} \quad(k=1, \cdots, r) .
\end{aligned}
$$

Let $T_{M}$ denote the matrix of $T$ relative to $\mathcal{D}$ if $T$ is a real-linear transformation of $G_{c}$. Finally, let the real numbers $C_{i j}^{k}$ be defined by $\left[X_{i}\right.$, $\left.X_{j}\right]=\sum_{k-1}^{2 r} C_{i j}^{k} X_{k}(i, j, k=1, \cdots, 2 r)$.

Clearly $T \in$ aut $G_{C}$ if and only if $\operatorname{det} T \neq 0$ and

(1) $T \iota=\iota T$,

(2) $\left[T X_{i}, T X_{j}\right]=T\left[X_{i}, X_{j}\right](i, j=1, \cdots, r)$.

The first condition states that

$$
T_{M}\left(\begin{array}{rr}
0 & E \\
-E & 0
\end{array}\right)=\left(\begin{array}{rr}
0 & E \\
-E & 0
\end{array}\right) T_{M}
$$

where $E$ is the $r \times r$ identity matrix. Condition (2) states that

$$
\sum_{\alpha, \beta=1}^{2 r} C_{\alpha \beta}^{k} a_{i}^{\alpha} a_{j}^{\beta}=\sum_{\gamma=1}^{2 r} C_{i j}^{\gamma} a_{\gamma}^{k} \quad(i, j=1, \cdots r),
$$

where $\left(a_{p}^{q}\right)=T_{M}(p, q=1, \cdots, 2 r)$. Thus aut $G_{C}$ is algebraic.

To prove that aut $G$ is algebraic, assume $\left\{X_{1}, \cdots, X_{r}\right\}$ chosen so as to be a subset of $G$. Then, clearly, $T \in$ aut $G$ if and only if $\operatorname{det} T \neq 0$ and

(1) $T \iota=\iota T$,

(2) $\left[T X_{i}, T X_{j}\right]=T\left[X_{i}, X_{j}\right](i, j=1, \cdots, r)$,

(3) $a_{i}^{j}=0$ if $j>r$.

Thus aut $G$ is algebraic. By a similar argument aut $G_{K}$ may be proved to be algebraic.

Lemma 2.3. $G^{*}, G_{K}^{*}, G_{C}^{*}$ are the components of the identity of aut $G$, aut $G_{K}$, aut $G_{c}$, respectively. 
Proof. $G, G^{*}, G_{C}^{*}$ are all semi-simple Lie algebras. The lemma now follows easily from the observations:

(1) If $\exp t X$ is an automorphism of $G_{C}$ (or $G$ or $G_{K}$ ) for all real $t$, then $X$ is a derivation of $G_{C}$ (or $G$ or $\mathscr{G}_{K}$ ).

(2) All the derivations of a semi-simple Lie algebra are inner.

LEMмA 2.4. Let $L$ be an algebraic Lie group containing $s$ $=\exp (-1)^{1 / 2} W$, where the matrix of $W$ relative to some base for the underlying (real) linear space is skew-symmetric. Then $\exp (-1)^{1 / 2} t W$ $\in L$ for all real $t$.

Proof. Choose a base $\mathcal{D}$ for the underlying linear space relative to which the matrix of $W=\operatorname{diag}\left\{(-1)^{1 / 2} \lambda_{1}, \cdots,(-1)^{1 / 2} \lambda_{n}\right\}, \lambda_{i}$ real. Relative to $\mathcal{D}$ the matrix of $s^{k}$ is diag $\left\{e^{-k \lambda_{1}}, \cdots, e^{-k \lambda_{n}}\right\}$.

If $f$ is a polynomial and $f\left(e^{-k \lambda_{1}}, \cdots, e^{-k \lambda_{n}}\right)=0$ for all positive integers $k$, then from the fact that $\lambda_{1}, \cdots, \lambda_{n}$ are real it can be inferred that $f\left(e^{t \lambda_{1}}, \cdots, e^{t \lambda_{n}}\right)=0$ for all real $t$.

Inasmuch as $L$ is algebraic, it is seen that $s^{k} \in L$ for all positive integers $k$ implies that $\exp (-1)^{1 / 2} t W \in L$ for all real $t$.

As seen in Lemma 2.1, $G=G \cap G_{K}+G \cap(-1)^{1 / 2} G_{K}$. Let $\mathcal{F}=G$ $\cap G_{K}$, let $\mathcal{F}^{*}=\{$ ad $X \mid X \in \mathcal{F}\}$, let $\mathcal{S}=G^{\prime}(-1)^{1 / 2} G_{K}$, and let $\mathcal{S}^{*}$ $=\{\operatorname{ad} X \mid X \in \mathcal{S}\}$. Let $F^{*}$ denote the Lie subgroup of $G_{C}^{*}$ corresponding to the real Lie subalgebra $\mathcal{F}^{*} \subset G^{*}$ and let $S^{*}=\left\{\exp X \mid X \in \mathcal{S}^{*}\right\}$. Let $\bigodot$ be a base for the complex linear space $G_{c}$ relative to which the matrix of ad $X$ is real skew-symmetric for all $X \in G_{K}$. Then $G_{C}^{*}$ is a subgroup of the orthogonal group relative to $\mathcal{C}$. Let $\tilde{F}^{*}$ denote the subgroup of $G^{*}$ whose matrices relative to $\mathcal{C}$ are real orthogonal.

Suppose now $g \in G^{*}$. Then $g=f \cdot s$ where $s=\exp (-1)^{1 / 2} W$, and relative to $\mathcal{C}$ the matrices of $f$ and $W$ are respectively real orthogonal and real skew-symmetric (cf. C. Chevalley, Lie groups, p. 15).

Lemma 2.5. In the above decomposition for $g, f \in \widetilde{F}^{*}$ and $s \in S^{*}$.

Proof. Let $\eta$ denote the conjugation of $G$ with respect to $G_{K}$, let $T_{M}$ denote the matrix of $T$ relative to $C$ if $T$ is a complex-linear transformation of $G_{C}$, and let $\eta^{*}(T)$ denote the complex-linear transformation of $G_{C}$ with matrix $\bar{T}_{M}$. Since $G=G \cap G_{K}+G \cap(-1)^{1 / 2} G_{K}$, $\eta(G)=G$. Moreover $\eta([X, Y])=[\eta(X), \eta(Y)]$ implies ad $\eta(X)(\eta(Y))$ $=\eta($ ad $X(Y))$ for $X, Y \in G_{C}$. On the other hand $\eta^{*}(\operatorname{ad} X)(\eta(Y))$ $=\eta(\operatorname{ad} X(Y))$. It follows that $\eta^{*}(\operatorname{ad} X)=\operatorname{ad} \eta(X)$ for $X \in G C$ and that $\eta^{*}\left(G^{*}\right)=G^{*}$ where $G^{*}=\{\operatorname{ad} X \mid X \in G\}$. Since $G^{*}$ consists of finite products of the type $\exp X_{1} \cdot \exp X_{2} \cdots \exp X_{8}$ where $X_{1}, \cdots, X_{s} \in G^{*}$, and since $\eta^{*}\left(\exp X_{1} \cdot \exp X_{2} \cdots \exp X_{s}\right)$ $=\exp \eta^{*}\left(X_{1}\right) \cdots \exp \eta^{*}\left(X_{s}\right)$ it is seen that $\eta^{*}\left(G^{*}\right)=G^{*}$. 
Consider now the relation $g=f \cdot s$. Then, $\eta^{*}(g)=\eta^{*}(f) \cdot \eta^{*}(s)=f \cdot s^{-1}$. Hence $s^{2}=\left(\eta^{*}(g)\right)^{-1} g$ is in $G^{*}$; that is, exp $2(-1)^{1 / 2} W \in G^{*}$.

Suppose $\mathcal{C}=\left\{Z_{1}, \cdots, Z_{r}\right\}$. Let $\mathcal{C}^{\prime}=\left\{Z_{1}, \cdots, Z_{r},(-1)^{1 / 2} Z_{1}, \cdots\right.$, $\left.(-1)^{1 / 2} Z_{r}\right\}$. Regard now $G_{C}$ as a linear space over the reals and denote by $T_{\mu}$ the matrix relative to $C^{\prime}$ of a real-linear transformation $T$ on $G_{C}$. Since the coefficients of $W_{M}$ are real, $W_{\mu}$ is the $2 r \times 2 r$ matrix

$$
\begin{gathered}
\left(\begin{array}{cc}
W_{M} & 0 \\
0 & W_{M}
\end{array}\right) . \\
{ }^{t} W_{\mu}=\left(\begin{array}{cc}
{ }^{t} W_{M} & 0 \\
0 & { }^{t} W_{M}
\end{array}\right)=\left(\begin{array}{cc}
-W_{M} & 0 \\
0 & -W_{M}
\end{array}\right)=-W_{\mu} .
\end{gathered}
$$

Consequently $W_{\mu}$ is a real skew-symmetric matrix. Applying Lemma 2.4 , it is seen that $\exp (-1)^{1 / 2} t \cdot 2 W$ belongs to aut $G$ for all real $t$ and thus, by Lemma 2.3 , to $G^{*}$. It follows that $(-1)^{1 / 2} W \in G^{*}$. Since $W_{M}$ is real, $W \in G_{K}^{*}$ and hence $(-1)^{1 / 2} W \in \mathcal{S}^{*}=G^{*} \cap(-1)^{1 / 2} G_{K}^{*}$. Consequently $s \in S^{*}$ and $f$, being in $G^{*}$, must be in $\tilde{F}^{*}$. Proof of Lemma 2.5 is now complete.

Let $S^{*}$ be endowed with its inner topology relative to $G^{*}$ (and $F^{*}$ with its topology qua Lie group).

LEMMA 2.6. $G^{*}=F^{*} \times S^{*}$ topologically. $F^{*}$ is a compact Lie subgroup and $S^{*}$ is topologically a Euclidean space.

Proof. Consider the one-to-one mapping $g \rightarrow(f, s)$ defined by: $g=f \cdot s$ where $f \in \tilde{F}^{*}, s \in S^{*}$ of $G^{*}$ onto the topological direct product $\tilde{F}^{*} \times S^{*}$.

It follows directly from Lemmas 2.2 and 2.3 and the fact that an algebraic Lie group is closed that $G^{*}$ and $G_{K}^{*}$ are closed in the group of all regular real-linear transformations on $G_{C}$ and a fortiori in the group of all regular complex-linear transformations on $G_{c} . \tilde{F}^{*}$, the set of all real orthogonal (relative to the base $\mathcal{C}$ ) complex-linear transformations in $G^{*}$, is clearly closed.

The mapping $g_{M} \rightarrow\left(f_{M}, s_{M}\right)$ is the restriction of the well known ${ }^{5}$ one-to-one bicontinuous mapping of the group of all regular complexlinear transformations of $G_{C}$ onto the topological direct product of the group of unitary matrices and the set of positive definite hermitian matrices (relative to $\mathcal{C}$ ). Furthermore, the exponential mapping of $S^{*}$ onto $S^{*}$ is the restriction to $S^{*}$ of the well known homeomorphism ${ }^{5}$ of the hermitian matrices onto the positive definite hermitian matrices. Since $G^{*}$ and $F^{*}$ are closed, their topologies as Lie groups

5 Cf. C. Chevalley, Lie groups, Princeton University Press, 1946, p. 16. 
and the given topology of $S^{*}$ are equivalent to their inner topologies relative to the group of all regular complex-linear transformations of $G_{c}$. It follows that $S^{*}$ is topologically a Euclidean space and that the mapping $g \rightarrow f \cdot s$ is a homeomorphism of $G^{*}$ onto $\tilde{F}^{*} \times S^{*}$.

Since $G^{*}$ is connected, $\widetilde{F}^{*}$ is connected. But the Lie algebra of $\tilde{F}^{*}$ is clearly $G^{*} \cap G_{K}^{*}$, the Lie algebra of the Lie subgroup $F^{*}$. Consequently $\widetilde{F}^{*}=F^{*}$, and $F^{*}$ is closed in the full complex-linear group on Gc. Inasmuch as $\left\{T_{M} \mid T \in F^{*}\right\}$ is a subgroup of the real orthogonal group (relative to base $C$ ), $F^{*}$ is compact. Proof of the lemma is now complete.

Let $D$ denote the center of the semi-simple group $G$. $D$ is discrete and $G^{*}=G / D$ topologically as well as algebraically, under the natural mapping $\zeta$ :

$g \in G \rightarrow$ automorphism of $G_{c}$ induced by $\Gamma_{g}$, where $\Gamma_{g}$ is the inner automorphism $x \rightarrow g^{-1} x g$ of $G$. Let $S=\{\exp X \mid X \in \mathcal{S}, \mathcal{S}$ $\left.=G \cap(-1)^{1 / 2} G_{K}\right\}$, and let $F$ denote the connected Lie subgroup of $G$ determined by $\mathcal{F}=G \cap G_{K}$.

$\zeta(\exp X)=\exp$ ad $X$ if $X \in G$. Inasmuch as the mappings $X \rightarrow \operatorname{ad} X$ and ad $X \rightarrow \exp$ ad $X$ are one-to-one if $X \in \mathcal{S}$, the mapping $X \rightarrow \exp X$ is one-to-one if $X \in \mathcal{S}$. Give $S$ the topology which makes the mapping $X \rightarrow \exp X$ of $S$ (endowed with its natural Euclidean topology) onto $S$ a homeomorphism and $F$ its topology qua Lie group. Clearly $\zeta$ maps $S$ homeomorphically onto $S^{*}$.

\section{LEMma 2.7. $G=F \times S$ topologically.}

Proof. Let $\widetilde{F}=\zeta^{-1}\left(F^{*}\right)$. The homogeneous space $G / \widetilde{F}$ is homeomorphic to $G^{*} / F^{*}=S^{*}$, a simply connected space. It follows that $\tilde{F}$ is connected, $\widetilde{F}=F$, and $D \subset F$.

Let $g \in G$. Since $\zeta(g)=f^{*} \cdot s^{*}$ where $f \in F^{*}$ and $s \in S^{*}, g=d \cdot f^{\prime} \cdot s$ where $f^{\prime} \in F, s \in S, d \in D$. But $D \subset F$; hence $g=f \cdot s$ where $f \in F, s \in S$.

Suppose that $f_{1} \cdot s_{1}=f_{2} \cdot s_{2}$ where $f_{1}, f_{2} \in F$ and $s_{1}, s_{2} \in S$. Then $\zeta\left(f_{1}\right) \zeta\left(s_{1}\right)=\zeta\left(f_{2}\right) \zeta\left(s_{2}\right)$ implies that $\zeta\left(s_{1}\right)=\zeta\left(s_{2}\right)$. Since $\zeta$ is one-to-one on $S, s_{1}=s_{2}$ and hence $f_{1}=f_{2}$, that is, the mapping

$$
\phi: \quad(f, s) \rightarrow g
$$

defined by $g=f \cdot s$ is a one-to-one mapping of $F \times S$ onto $G$.

We now prove that $\phi$ is a homeomorphism. Inasmuch as the exponential mapping of a Lie algebra (endowed with its natural Euclidean topology) into a Lie group is a continuous mapping, and inasmuch as group multiplication is continuous, the mapping $\phi$ is continuous.

Let $U, V$ be connected neighborhoods in $F, S$ respectively. Since $\zeta$ 
is a homeomorphism on $S, \zeta(V)$ is a connected open set of $S^{*}$. Since $F$ and $F^{*}$ are closed subgroups of $G$ and $G^{*}$ respectively, it follows that their topologies qua Lie groups are equivalent to their inner topologies relative to $G$ and $G^{*}$ respectively. Consequently $\zeta(U)$ is a connected open set of $F^{*}$ and $\zeta(\phi(U \times V))$ is a connected open set of $G^{*}$.

$$
\zeta(\phi(U \times V))=\zeta(U \cdot V)=\zeta(U) \cdot \zeta(V) .
$$

Hence by selecting $U$ and $V$ sufficiently small, $\zeta(\phi(U \times V))$ can be made arbitrarily small. Since $G$ is a covering space of $G^{*}$ under $\zeta$, $\zeta^{-1}(N)$ is a union of disjoint connected open sets if $N$ is a sufficiently small connected open set. Inasmuch as $\phi(U \times V)$ is connected, it is a (connected) open set of $G$ if $U$ and $V$ are sufficiently small. The mapping $\phi$ is therefore open.

LEMmA 2.8. $\mathfrak{F}^{*}=\mathcal{L}^{*} \oplus \mathcal{A}^{*}$ (directly) where $\mathcal{L}^{*}$ and $\mathcal{A}^{*}$ are respectively semi-simple and abelian ideals of $\mathcal{F}^{*}$. Let $L^{*}, A^{*}$ be the Lie subgroups of $F^{*}$ determined by $\mathcal{L}^{*}, A^{*}$ respectively. Then $L^{*}$ and $A^{*}$ are compact.

Proof. Consider the adjoint group of $F$ acting on $\mathcal{F}$. Since $F^{*}$ is compact, the adjoint group of $F$ (a homomorphic image of $F^{*}$ ) is compact and is therefore completely reducible, that is, if the linear transformations of the adjoint group keep a subspace invariant, they keep invariant a complementary subspace. It follows that $\mathcal{F}$ is the direct union of minimal ideals. Consequently $\mathcal{F}=\mathcal{L} \oplus \mathcal{A}$ (directly) where $\mathcal{L}$ and $\mathcal{A}$ are respectively semi-simple and abelian ideals of $\mathcal{F}$. Let $\mathcal{L}^{*}, A^{*}$, be the images of $\mathcal{L}, \mathcal{A}$ respectively under the adjoint representation of $G$. Then $\mathcal{F}^{*}=\mathcal{L}^{*} \oplus \mathcal{A}^{*}$ (directly).

Since $\mathcal{L}^{*}$ contains no abelian ideals, $\mathcal{A}^{*}$ can clearly be characterized as the maximum abelian ideal of $\mathcal{F}^{*}$. It follows that $A^{*}$ is the maximum connected normal abelian Lie subgroup of $F^{*}$. Since $\bar{A}^{*}$, the topological closure of $A^{*}$, is a connected normal abelian Lie subgroup, $\bar{A}^{*}=A^{*}$, that is, $A^{*}$ is a closed subgroup of $F^{*}$ and is thus compact.

Consider now $F^{*} / A^{*}$. This is a compact Lie group whose Lie algebra is isomorphic to $\mathcal{F}^{*} / \mathcal{A}^{*}=\mathcal{L}^{*}$. Since there is a compact Lie group with Lie algebra isomorphic to $\mathcal{L}^{*}$, by a theorem of Weyl $1^{6}$ any Lie group whose Lie algebra is isomorphic to $\mathcal{L}^{*}$ is compact. Consequently $L^{*}$ is compact.

TheOREM 2. $G=K \times E$ topologically, where $K$ is a maximal compact subgroup and $E$ is topologically a Euclidean space.

- H. Weyl, loc. cit. p. 170. 
Proof. As was seen in the proof of Lemma $8, \mathcal{F}=\mathcal{L} \oplus \mathcal{A}$ where $\mathcal{L}$ is semi-simple and $\mathcal{A}$ abelian. Let $L, A$ be the Lie subgroups of $F$ corresponding to $\mathcal{L}, \mathcal{A}$ respectively, $\zeta(L)=L^{*}$ and $\zeta(A)=A^{*}$. $A$, being the component of the identity of the closed subgroup $\zeta^{-1}(A)$, is closed.

Since $\mathcal{L} \cong \mathcal{L}^{*}$ and $L^{*}$ is compact, by Weyl's theorem $L$ is compact.

Let $T \times V$ be a decomposition of the connected abelian Lie group $A$ into the direct product of a toroidal group $T$ and a vector group $V$. $V$ is closed in $A$ and hence in $F$.

Set $K=L T$, that is, $K=\{l t \mid l \in L, t \in T\} . K$ is a compact normal subgroup of $F$ and $F=K V . K$ and $V$ being closed subgroups of $F$, $K \cap V$ is both compact and a subgroup of $V$; it follows that $K$ and $V$ intersect in only the identity of $F$. Inasmuch as $K$ and $V$ are closed normal subgroups of $F, F=K \times V$ topologically as well as algebraically. Finally, let $E=V \cdot S$. Clearly $E$ is topologically the direct product of two Euclidean spaces and is hence itself Euclidean. It may now be asserted that $G=K \times E$ topologically.

HARVARD UNIVERSITY AND

InSTITUTE FOR AdVANCED STUdY 